\title{
obituary
}

\section{Benjamin Lee}

Professor Benjamin W. Lee died in an automobile accident on 16 June 1977. Director of Theoretical Physics at Fermi National Accelerator Laboratory and Professor of Physics at the University of Chicago Enrico Fermi Institute, Ben Lee was at the age of 42 deeply engaged in scientific activity at the forefront of high energy physics.

Born in Seoul, Korea, Ben Lee emigrated to the United States in 1956 and obtained citizenship in 1968 . He studied at Miami University (Oxford, Ohio), the University of Pittsburgh and the University of Pennsylvania, where he was awarded a doctorate in 1960. His earliest work dealt primarily with dispersion relations and related techniques in the analysis of strong interaction phenomena, but he also had an early interest in Lagrangian symmetries and spontaneous symmetry breakdown, which ultimately became the focal points of his research.

By 1964 Ben Lee was a leading contributor to the study of the transformation properties of weak interactions with respect to strong interaction symmetries, and the intimately related question of the full symmetry properties of the strong interactions themselves. Through such studies the relevance of a spontaneously broken chiral symmetry gradually became apparent, and Lee contributed significantly to the construction and study of Lagrangian field theories which realise this property. His work demonstrating the renormalisability of these theories provided an encouraging model for later developments which led in 1971 to the establishment of renormalisability of spontaneously broken gauge theories. Following this breakthrough, Lee's work on the formal demonstration of renormalisability of gauge theories and on the elaboration of calculation techniques was of major importance in the further development of this field which has taken a dominant position in our present thinking.

As soon as the relevance of gauge theories for weak interactions began to appear highly probable, Ben Lee turned to the study of their practical implications, both for the newly discovered neutral current interactions and for the previously established properties of charged curnent interactions.
The use of new theoretical developments allowed a confrontation with experiment which placed severe restrictions on the possible choice of gauge models. It became increasingly clear that a viable model should incorporate charmed partioles which had been postulated before achievements in renormalisation made such an inference compelling. Lee devoted considerable study to the expected properties of these particles and the possibilities for their experimental detection.

Along with the experimental discovery of charm, there has been a continuous experimental and theoretical evolution in the field of both weak and strong gauge theories, in which Ben Lee continued to participate fully. His rare combination of a familiarity with formal mathematical techniques and a close touch with experimenters and experimental results kept him at the forefront of many different aspects of these developments. At the same time he continued to reflect on the outstanding problems related to his earlier work on weak interaction symmetries and was quick to apply new developments to the clarification of old problems. $\mathrm{He}$ encouraged precision experiments designed to resolve longstanding questions as well as pioneering experimentation on the new phenomena.

Ben Lee's decision to remain permanently as head of the Fermilab theory group was not an easy one for him. He felt that at a university he would be freer to pursue fundamental theoretical questions. Yet while continuing to participate in the fast pace of theoretical research, he took extremely seriously the responsibilities of his position at Fermilab. On the one hand he attracted theoretical physicists of the highest quality as visitors to the laboratory and was in the process of putting together a small but strong nucleus of resident theorists. On the other hand he was deeply involved in laboratory policy concerning its present and future experimental programme, a job which he undertook with singular conscientiousness, endeavouring to learn the fine points of experimental techniques.

Before joining the Fermilab staff in 1973, Benjamin Lee was Professor of Physics at the State University of New York at Stony Brook. In addition to his responsibilities at Fermilab he served on the scientific policy com- mittees for several major laboratories. $\mathrm{He}$ was the recipient of a number of prestigious scientific fellowships and had held appointments at many institutions in the United States and abroad. Perhaps his most meaningful experience in recent years was a visit to his native Korea where he was struck by the developing prospects for scientific research and found himself "working six days a week on upgrading graduate education in basic sciences" at the Seoul National University.

Ben Lee shared a close family life with his wife Marianne and teen-aged son and daughter at their home in Glen Ellyn, Illinois. Whenever possible they combined business with pleasure on trips to Aspen in Colorado, Europe and the Far East. An immeasurable personal tragedy for his family, Benjamin Lee's untimely death is a deep loss for the entire high energy physics community.

Mary K. Gaillard

\section{Fred Hardy}

Professor Fred HARdy, CBE, who died at St Augustine, Trinidad, in May at the age of 88 was the doyen of British tropical soil scientists. During his professional life the study of the soil developed from a subject that had little impact on practical agriculture to a discipline that has made major contributions to agricultural development, and Professor Hardy played a notable role in this transformation for tropical soil studies.

Almost his whole adult life was spent in the West Indies and Central America. His first appointment after leaving Cambridge University, was as lecturer in natural and agricultural sciences at Harrison College, Barbados, in 1911. He returned to England in 1917 towards the end of the First World War, then after the war spent a year at the School of Agriculture at Cambridge and then went back in 1920 to the West Indies as soil chemist with the Imperial Department of Agriculture, which was centred at $\mathrm{St}$ Augustine. This later became the Imperial College of Tropical Agriculture, and he became its first Professor of Chemistry and Soil Science. After his retirement in 1956 he served as Professor of Soil Science in the Inter-American Institute of Agricultural Sciences at Turrialba, Costa 